This is the author's final, peer-reviewed manuscript as accepted for publication. The publisher-formatted version may be available through the publisher's web site or your institution's library.

\title{
Anti-breast cancer agents, quinolines, targeting gap junction
}

Julie Bernzweig, Brian Heiniger, Keshar Prasain, Jianyu Lu, Duy H. Hua and Thu A. Nguyen

\section{How to cite this manuscript}

If you make reference to this version of the manuscript, use the following information:

Bernzweig, J., Heiniger, B., Prasain, K., Lu, J., Hua, D. H., \& Nguyen, T. A. (2011). Antibreast cancer agents, quinolines, targeting gap junction. Retrieved from http://krex.ksu.edu

\section{Published Version Information}

Citation: Bernzweig, J., Heiniger, B., Prasain, K., Lu, J., Hua, D. H., \& Nguyen, T. A. (2011). Anti-breast cancer agents, quinolines, targeting gap junction. Medicinal Chemistry, 7(5), 448-453.

Copyright: ( 2011 Bentham Science Publishers Ltd.

Digital Object Identifier (DOI):

Publisher's Link: http://www.benthamdirect.org/pages/b_bypublication.php

This item was retrieved from the K-State Research Exchange (K-REx), the institutional repository of Kansas State University. K-REx is available at http://krex.ksu.edu 


\section{Anti-breast Cancer Agents, Quinolines, Targeting Gap Junction}

Julie Bernzweig ${ }^{1}$, Brian Heiniger ${ }^{1}$, Keshar Prasain ${ }^{2}$, Jianyu Lu $^{2}$, Duy H. Hua ${ }^{2}$ and Thu A. Nguyen ${ }^{1}$

${ }^{1}$ Department of Diagnostic Medicine/Pathobiology, Kansas State University, Manhattan, KS 66506

${ }^{2}$ Department of Chemistry, Kansas State University, Manhattan, KS 66506

Corresponding Author: Thu Annelise Nguyen

E-mail: tnguyen@vet.ksu.edu

Phone: 785-532-4429

Fax: $785-532-4039$

\section{Abstract}

Cancer cells exhibit many defects in cell communication that contribute to the loss of tissue homeostasis (excess cell proliferation, invasion, and metastasis). The process of cancer formation causes a disruption in cell homeostasis, affecting the ability to respond to extracellular signals, as well as triggering some intracellular events which alter gap junctional intercellular communication (GJIC). Previous research has shown that the first two generations of substituted quinolines have anti-cancer effects on human breast cancer cells. This report presents the synthesis and bioactivities of the third generation of substituted quinolines. Scrape load/dye transfer studies showed that $100 \mathrm{nM}$ of PQ15, a third generation of substituted quinolones, can cause a 4.5-fold increase of gap junction activity in T47D breast cancer cells. Furthermore, a significant decrease of cell proliferation and viability was observed in the presence of $200 \mathrm{nM}$ PQ15 compared to control. The expression of $\alpha$-survivin was reduced to $<18 \%$ in the treatment of $100 \mathrm{nM} \mathrm{PQ15}$ compared to control without treatment or solvent. Alpha-survivin expression is upregulated in human cancers and associated with resistance to chemotherapy, suggesting that $\alpha$-survivin prolongs the survival of cancer cells. Thus, it has 
been shown that substituted quinolines stimulate gap junction activity, decrease alpha survivin expression, and subsequently inhibit cancer cell growth. Our findings demonstrate that PQ15 has a promising role in exerting anti-cancer activity in human breast cancer cells.

\section{Introduction}

The influence of hormones on the development and differentiation of the mammary gland has been described in numerous publications in regard to the role of estrogen in ductal growth, progesterone in lobulo-alveolar development, and prolactin in milk secretion [1]. However, the mechanism by which this local control of growth and differentiation of the constituent epithelial cell types occur is unclear. Some candidates are locally-acting growth factors, epithelial-stromal interactions, and direct cell-cell communication [2]. Direct cell-cell communication such as gap junctions may be modulators underlying cellular differentiation and also co-ordinates of cellular function in the mammary gland.

Numerous reports have been confirmed that gap junctions are frequently decreased or absent in cancer cells [3]. For instance, co-culture of tumor cells with normal cells, or with connexin-overexpressing cells, results in growth retardation of neoplastic component by the establishment of functional communication as observed with dye transfer [4]. This retardation of tumor growth could be prevented by co-culturing transformed cells with junctional competent normal cells transfected with a connexin-specific antisense reagent [5]. Thus, these results indicate that signals from adjacent normal cells can reverse a malignant phenotype and that their failure to accomplish this is due to a lack of GJIC.

The loss of GJIC during carcinogenesis may not result from a direct disruption of a specific connexin gene or mRNA levels. The cellular oncogenes downregulate GJIC by phosphorylating gap junction proteins, connexin 32 (Cx32) and connexin $43(\mathrm{C} \times 43)$. In the case of $\mathrm{Cx} 43$, the phosphorylation of $\mathrm{C}$-terminal serine residues results directly from protein kinase $\mathrm{C}$ (PKC) [6]. Furthermore, evidence demonstrated that PKCs inhibit gap junction channels by 
inhibiting gap junction assembly and channel gating [7]. PKC a overexpression has been shown to cause tumor growth, and tumor resistance to cytotoxic chemotherapy. We have shown that PKC $\alpha$ is highly expressed in MCF-7 breast cancer cells and stimulation of PKC $\alpha$ in these cells caused a further inhibition of gap junction activity [8].

Several studies involving breast cancer patient tissue samples show a loss of overall connexin protein expression or a relocalization of the connexin to intracellular compartments [911]. Multiple in vitro studies confirm the loss of connexin and/or GJIC in breast cancer cells. Early studies showed that connexin 26 (Cx26) and Cx43 were downregulated at the mRNA level in primary cells derived from human breast tumors [11] as well as in breast cancer cell lines [10]. Furthermore, extensive studies involving the reconstitution of connexin expression in GJIC-deficient breast cancer cell lines have shed some light on their role in cell growth control and differentiation. Re-expression of $\mathrm{Cx} 43$ in MDA-MB-435 breast cancer cells resulted in growth suppression, a regained ability to differentiate into three-dimensional (3D) structures in the presence of a basement membrane and, importantly, reduction of tumor growth in mice [12]. Interestingly, increasing cell-cell communication in Cx43 overexpressing tumor cells enhances drug sensitivity [13]. Cancer cells are characterized by the lack of growth control, by the inability to terminally differentiate and by resistance to apoptosis. Additionally, connexins have shown to have a GJIC-independent role in the regulation of apoptosis, as suggested from the correlation of intracellular Cx26 and Cx43 expression with the presence of the proapoptotic factor Bak [9]. Previous work demonstrated that first and second generations of substituted quinolines can activate apoptotic protein, caspase 3 , in addition to upregulation gap junction activity in breast cancer cells [14]. This supports the idea that reversed process such as increase or gain of GJIC in cancer cells can drive toward apoptosis of breast cancer cells. Furthermore, transfection of gap junction genes, connexins, into GJIC-deficient tumor cells can restore GJIC, growth control and reduce tumorigenicity [15]. The modulation can either downregulate GJIC and lead to tumor promotion or it can upregulate GJIC and lead to 
suppression of the initiated cells [16]. Hence, increasing gap junction activity or enhancing GJIC in tumor cells provides the means to enhance antineoplastic therapies.

Based on previous synthesis, we designed and synthesized structurally similar analogs with enhanced bioactivity and improved drug characteristics, affording lead compounds suitable for preclinical development. Structure-activity relationship (SAR) studies allowed us to optimize the potency of these compounds through iterative medicinal/combinatorial chemistry, molecular modeling and in vitro testing. Therefore, this report demonstrates that the third generation of subsitituted quinoline, PQ15 (Figure 1), can increase gap junction activity in T47D breast cancer cells and significantly cause a decrease in the expression of $\alpha$-survivin.

\section{Materials and Methods}

Synthesis of PQs: A small library of PQ1 - PQ5 was first synthesized and PQ1, containing an aminopropyl side chain at N1', was found to be the most active compound in both GJIC enhancement and anticancer activities among PQ1 - PQ5 (PQ2 - PQ5 are inactive and the data were not shown). Hence, analogs of PQ1, PQ6 - PQ11, were synthesized by varying the N1' substituent and studied their anticancer activities. Fortuitously, PQ11 containing a furanylmethyl moiety (maintains a similar distance as that of the aminopropyl moiety of PQ1) at $\mathrm{N} 1$ ' side chain shows potent anticancer activity with an $\mathrm{IC}_{50}$ value of $15.6 \mathrm{nM}$.

The syntheses of PQ1 and PQ11 have been reported by us [17] and the synthesis of PQ15, 6-methoxy-4-methyl-8-[(4-quinolinylmethyl)amino]-5-(3-trifluoromethylphenyloxy)quinoline, followed a similar synthetic sequence as that of PQ11. Hence, reductive amination reaction of 6-methoxy-4-methyl-8-amino-5-(3-trifluoromethylphenyloxy)quinoline and 4quinolinecarboxaldehyde (Plobeck et al. 2000) in methanol followed by sodium cyanoborohydride.

Cell line and cell culture. T47D human breast cancer cell line was purchased from American Type Culture Collection (Manassas, VA). Cells were grown in RPMI medium supplemented 
with $10 \%$ fetal bovine serum (Atlanta Biologicals, Lawrenceville, GA), 10\% antibioticactinomycotic at $37^{\circ} \mathrm{C}$ with $5 \% \mathrm{CO}_{2}$ in $75 \mathrm{~cm}^{2}$ flasks.

Gap Junction Activity. For scrape load/ dye transfer (SL/DT) assay, cells were grown to $90 \%$ confluency on cover slips, dosed with 10, 100, 200, and $1000 \mathrm{nM}$ of PQ15 for 3 hours. After that cells were washed three times with PBS. The $2.5 \mu \mathrm{l}$ of $1 \%(\mathrm{w} / \mathrm{v})$ Lucifer yellow and $.75 \%$ (w/v) of Rhodamine dextran was mixed and added in the center of the coverslip. Two cuts crossing each other in the center of the coverslip were made. After five minutes, cells were washed three times with PBS and incubated at $37^{\circ} \mathrm{C}$ in tissue culture media for 20 minutes. The cells were then washed with PBS three times and fixed in $2.5 \%$ paraformaldehyde for 10 minutes. Cells were mounted on a slide, sealed and visualized under a fluorescence microscope at $4 \mathrm{X}$ objective.

Western Blot Analysis. Cells were grown in serum supplemented RPMI media until they were $90 \%$ confluent in $75 \mathrm{~cm}^{2}$ flasks. Cells were dosed with $0,10,100$, and $1000 \mathrm{nM}$ of PQ15 for 24 hours. Cells were washed three times with cold PBS and then were harvested using lysis buffer (20 mM Tris pH 7.5, 0.5 mM EDTA, 0.5 mM EGTA, $0.5 \%$ Triton X-100) with 1:1000 dilution of protease inhibitors (Sigma-Aldrich, Saint Louis, MO). Cell lysate was sonicated and centrifuged at $13,000 \mathrm{rpm}$ for 30 minutes at $4^{\circ} \mathrm{C}$. Twenty-five $\mu \mathrm{g}$ of whole cell extract was resolved by $10 \%$ SDS-polyacrylamide gel electrophoresis (PAGE) and transferred to nitrocellulose membrane (Midwest Scientific, Saint Louis, MO). Nitrocellulose membrane was blocked in $5 \%$ milk for an hour at room temperature and then incubated with polycolonal rabbit $\alpha$-survivin, 1:500 (Santa Cruz Biotechnologies, Santa Cruz, California), monoclonal mouse Cx43, 1:500 (Fred Hutchinson, Seattle, Washington), rabbit actin, 1:1,000 (Sigma-Aldrich, Saint Louis, MO). Western blots were detected by enhanced chemiluminescence detection reagents (Pierce, Rockford, Illinois, USA).

Trypan Blue Excision. Cell viability was measured using the trypan blue excision method. $1 \mathrm{x}$ $10^{4}$ T47D breast cancer cells were treated with various concentrations of PQ15 for 48 hours. A 
cell suspension was mixed with trypan blue dye and then visually examined for viable cells by the Cellometer Auto T4 (Nexcelom). The graphical presentation includes at least three independent samples.

Statistical analysis: The level of significance (see ${ }^{*}$ in figure legends) was considered at $p<$ 0.05 using Student's $t$ test analysis. All data are presented as mean \pm S.D. of at least three independent experiments from different batches of cultures.

\section{Results and Discussion}

Approximately one in eight women is affected by breast cancer, which is the leading cause of death in females between the ages of 40 and 50 years in the Western world [18]. Antibreast cancer agents include tamoxifen (Nolvadex ${ }^{R}$; partial antiestrogens), fulvestrant (Faslodex ${ }^{\mathrm{R}}$; pure antiestrogens), and Lapatinib $\left(\right.$ Tykerb $\left.^{\mathrm{R}}\right)$, as well as of aromatase inhibitor, Femara $^{\mathrm{R}}$. There are several ongoing studies including pre-clinical and clinical trials with small drug-like molecules. Studies on gap junction enhancers onto breast cancer are limited. The study goal is to synthesize small molecules that specifically activate GJIC activity and inhibit cancer cell growth. Previously, a small library of novel quinolines, PQ1-PQ5, was first synthesized and PQ1, containing an aminopropyl side chain at N1', was found to be the most active compound in both GJIC enhancement and anticancer activities (14). Hence, analogs of PQ1, PQ6 - PQ11, were synthesized by varying the N1' substituent and studied their anticancer activities (20). $\quad$ PQ15, 6-methoxy-4-methyl-8-[(4-quinolinylmethyl)amino]-5-(3trifluoromethylphenyloxy)-quinoline, followed a similar synthetic sequence as that of PQ11, was synthesized as the third generation of this quinoline class (Figure 1). Hence, reductive amination reaction of 6-methoxy-4-methyl-8-amino-5-(3-trifluoromethylphenyloxy)quinoline and 4-quinolinecarboxaldehyde (21) in methanol followed by sodium cyanoborohydride.

A third generation of substituted quinolines was designed and their enhancement of GJs activities and killing of human breast cancer cells was demonstrated. PQ15 was tested on the 
effect of GJIC activity in T47D breast cancer cells (Figure 2A-H). The results demonstrated that $100 \mathrm{nM}$ of PQ15 showed a significant increase in gap junction activity compared to controls, without PQ15 treatment, using scrape load/dye transfer assay (Figure 2D). However, $1000 \mathrm{nM}$ PQ15 did not significantly increase the gap junction activity (Figure 2F). This may due to the cytotoxic effect at a higher concentration. Furthermore, the increase of gap junction activity by 100 and 200 nM PQ15 can be inhibited by 12-O-tetradecanoylphorbol-13-acetate (TPA) (Figure 2G-H). Previously, TPA has been shown to activate protein kinase $\mathrm{C}(\mathrm{PKC})$ and subsequently phosphorylate Cx43 on serine 262 and serine 268 [19]. The distance of dye transfer from section cut to the farthest cells with dye was measured. A graphical presentation of three experiments indicates that $100 \mathrm{nM}$ PQ15 causes a 4.5 -fold increase in distance of dye transfer compared to control (Figure 2I); however, $200 \mathrm{nM}$ TPA reduces this effect of $100 \mathrm{nM}$ PQ15 from 4.5-fold to 1.3-fold relative to control of gap junction activity. Interestingly, $200 \mathrm{nM}$ TPA was not sufficient to reduce gap junction activity of 200 nM PQ15 with 2.4-fold increase of gap junction activity. This suggests that PQ15 increases gap junction activity in part through TPAphosphorylating connexin 43 pathway. Comparison to previous analogs of $P Q$ series, PQ15 is less potent its precursors $[14,20]$. This indicates that the structure of highly quinolines in PQ15 is affecting the function of GJIC enhancement in T47D cells.

The effect of PQ15-upregulated gap junction activity in T47D cell proliferation was examined. T47D cells were treated with $0,10,100,200$ and $1000 \mathrm{nM} \mathrm{PQ15}$ for 48 hours. A graphical presentation of three experiment results is presented in Figure 3. The results show that $10 \mathrm{nM}$ PQ15 is not sufficient to affect cell proliferation; however, 100, 200 and $1000 \mathrm{nM}$ PQ15 cause a significant decrease of $18 \%, 30 \%$, and $32 \%$, respectively, in cell proliferation compared to control or DMSO. This suggests that $200 \mathrm{nM}$ PQ15 can cause an increase in gap junction activity and subsequently can decrease cell proliferation of T47D cells. Furthermore, cell viability of T47D cells was also performed (Figure 4). A $100 \mathrm{nM}$ or $200 \mathrm{nM}$ PQ15 for 48 hours reduces $16 \%$ of T47D cell viability and $1000 \mathrm{nM}$ PQ15 has a greater effect with $36 \%$ 
decrease in cell viability compared to control or DMSO. This supports the previous observation where 1000 nM PQ15 has less gap junction activity than 100-200 nM PQ15. The loss of cellcell interaction is due to cytotoxic effect of PQ15 at $1000 \mathrm{nM}$.

Furthermore, whole cell extract of PQ15 treatment was analyzed for the changes in gap junctional proteins, connexins. Cells were treated with 10, 100, and 1000 nM PQ15 for 24 hours. Western blot analysis was performed against Cx43 (Figure 5A). The results show that PQ15 has no effect on the total Cx43 expression; however, PQ15 causes a decrease in the phosphorylation of Cx43. This suggests that the increase of gap junction activity by PQ15 is due to the inhibition of phosphorylation of Cx43. The result also confirms that PQ15 and TPA mediate gap junction activity through the phosphorylation of Cx43.

Alpha-survivin expression is upregulated in human cancers and associated with resistance to chemotherapy, suggesting that $\alpha$-survivin prolongs the survival of cancer cells. The next step was to determine whether PQ15 affects the survival pathway of T47D cells. Cells were treated with 0, 10, 100, and 1000 nM PQ15 for 24 hours (Figure 5B). The results show that 100-1000 nM PQ15 can cause a significant decrease of $\alpha$-survivin expression compared to control or DMSO. This supports that PQ15 inhibits the expression of $\alpha$-survivin, preventing cell survival, and subsequently decreases the cell viability of T47D cells.

In conclusion, the anti-cancer activity of PQ15 in T47D cells is less potent as that of PQ1 and PQ11. The results imply that replacement of the furan moiety of the appendage at C8amine decreases potency. However, it is important to find that an addition of a quinoline nucleus to the aminoquinoline $P Q$ does not impede the anticancer activity. From an immunoprecipitation assay, it was found that $P Q$ disrupts the interaction of $\mathrm{Cx} 43$ and Nedd4, an E3 ubiquitin ligase, resulting in the inhibition of phosphorylation of $\mathrm{Cx} 43$ by protein kinase $\mathrm{C}$ and maintaining GJIC (data not shown). This suggests that PQ blocks the binding of Nedd 4 with Cx43, and the binding site appears to accommodate relatively larger groups at C8 appended alkylamino moiety including an extra quinoline nucleus. The results imply that modifications of 
the C8 appendage can be carried out for future improvement of anticancer potency as well as pharmacokinetics of this class of compounds.

\section{Summary}

Breast cancer cells have reduced GJIC and a high rate of cell proliferation in comparison to normal mammary cells. In the current study, the rationale is to synthesis a third generation of gap junction enhancers and examines the anti-cancer activity. Newly synthesized quinoline, PQ15, specifically enhances gap junction activity through TPA-mediated gap junction pathway. An increase of PQ15-induced gap junction activity causes a decrease in cell proliferation and cell viability. Overall, the findings provide a promising direction that third generation of substituted quinolines enhances gap junction activity by preventing the phosphorylation of connexn 43 and inhibits the expression of $\alpha$-survivin in T47D breast cancer cells.

Figure 1. Chemical Structures of PQ1, PQ11, and PQ15<smiles>COc1cc(NCCCN)c2nccc(C)c2c1Oc1cccc(C(F)(F)F)c1</smiles>

PQ1<smiles>COc1cc(NCc2ccco2)c2nccc(C)c2c1Oc1cccc(C(F)(F)F)c1</smiles>

PQ11<smiles>COc1cc(NCc2ccnc3ccccc23)c2nccc(C)c2c1Oc1cccc(C(F)(F)F)c1</smiles>

PQ15 

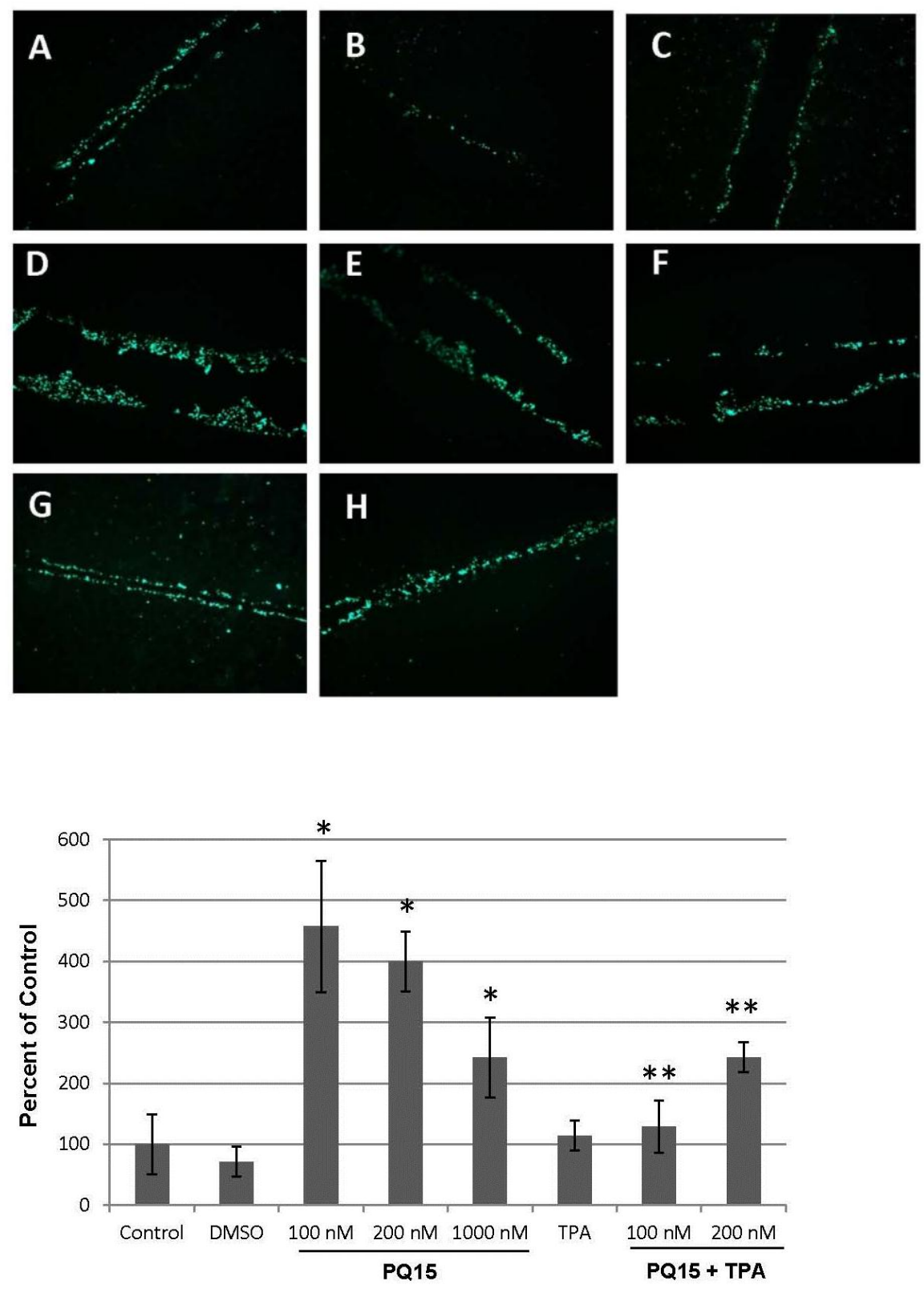

Figure 2. Gap Junction Activity of PQ15 in T47D breast cancer cells. T47D cells were treated with $0,100,200$, and $1000 \mathrm{nM}$ PQ15 for 3 hours (A, D, E and F). Cells were treated with 200 nM TPA alone (C), 100 nM PQ15 and 200 nM TPA (G), or 200 nM PQ15 and 200 nM TPA (H) for 3 hours. Controls are media alone (A) or solvent, DMSO (B). Scrape load/dye transfer assay was performed as described in Materials and Methods. Lucifer yellow was used as a gap junctional dye and rhodamine-dextran was used to mark the cutting site. Fluorescence green indicates the passages of dye from the cutting site, showing increase of GJIC. Graphical presentation of three experiments shows the distance of dye transfer of T47D cells. 


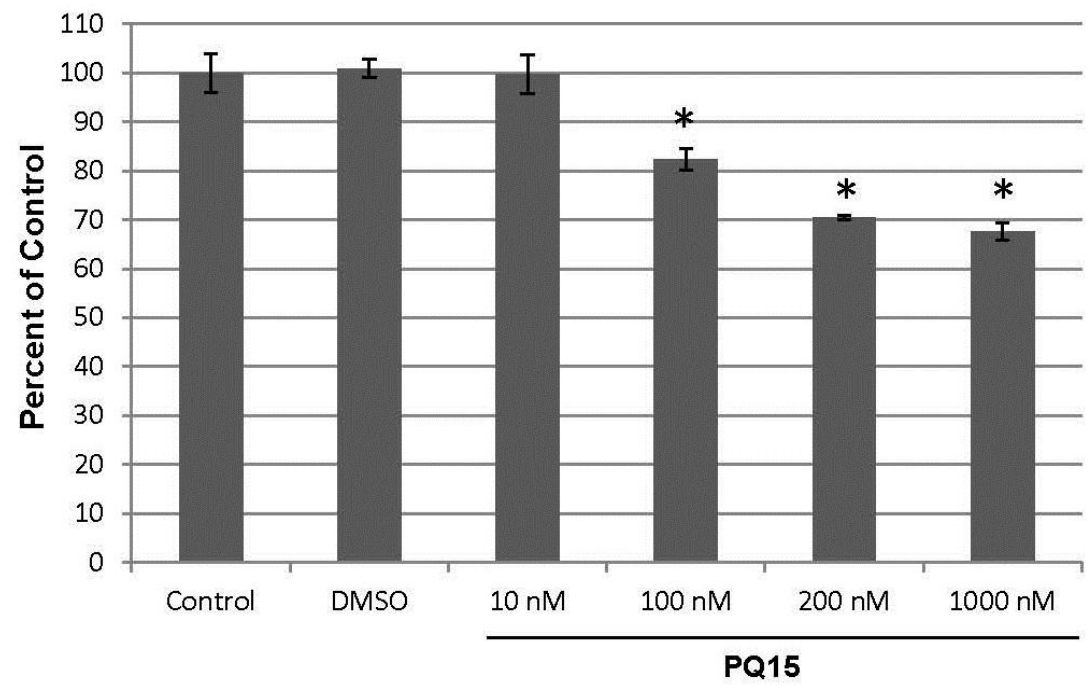

Figure 3. Effect of PQ15 on Cell Proliferation. $1 \times 10^{4}$ T47D breast cancer cells were treated with $0,10,100,200$, and $1000 \mathrm{nM}$ of PQ15 for 48 hours. Controls are media alone and DMSO as solvent. Total number of cells was counted and expressed in percent of control. The graphical presentation of three independent samples was calculated and indicated with a statistical significance, ${ }^{*} p<0.05$.

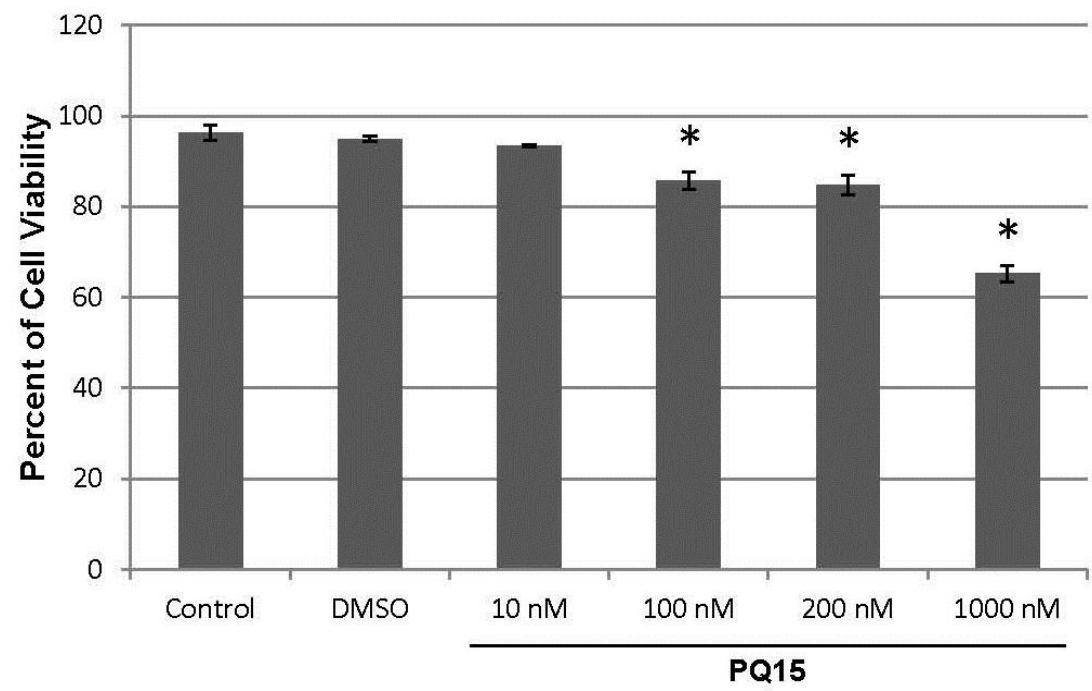

Figure 4. Effect of PQ15 on Cell Viability. $1 \times 10^{4}$ T47D breast cancer cells were treated with various concentrations of PQ15 for 48 hours. A cell suspension was mixed with trypan blue dye and then visually examined viable cells. The graphical presentation of three independent samples was measured with a statistical significance, ${ }^{\star} p<0.05$. 
A.
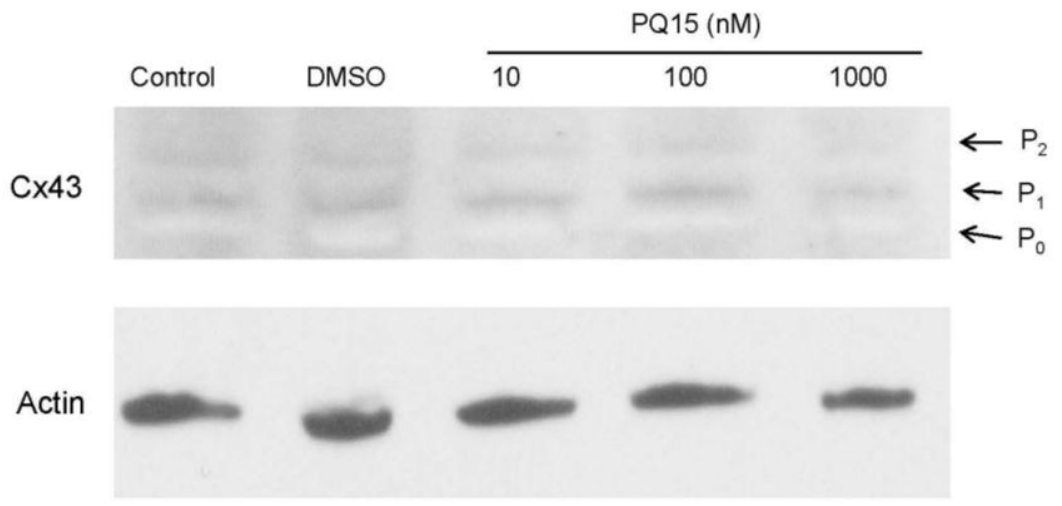

B.

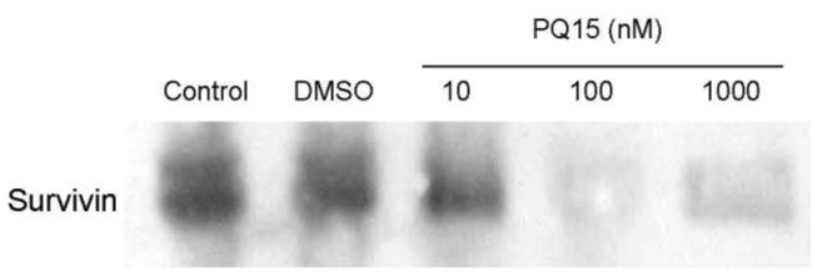

Figure 5. Effect of PQ15 on Connexin 43 and Survivin Expression. T47D cells were treated with 0, 10, $100 \mathrm{nM}$, and $1000 \mathrm{nM} \mathrm{PQ15}$ for 24 hours. Treatment with DMSO was used as control. Western blot analysis was performed. Nitrocellulose membrane was blotted with the active form of $\mathrm{Cx} 43$ (43 kDa) and a-survivin (16.5 kDa) antibodies. Actin acts as loading control. $\mathrm{P}_{0}=$ unphosphorylation, $\mathrm{P}_{1}=$ phosphorylation, $\mathrm{P}_{2}=$ multiple phosphorylations

\section{References}

1. Topper, Y.J. and C.S. Freeman, Multiple hormone interactions in the developmental biology of the mammary gland. Physiol Rev, 1980. 60(4): p. 1049-106.

2. Deome, K.B., et al., Development of mammary tumors from hyperplastic alveolar nodules transplanted into gland-free mammary fat pads of female $\mathrm{C} 3 \mathrm{H}$ mice. Cancer Res, 1959. 19(5): p. 515-20.

3. Loewenstein, W.R. and Y. Kanno, Intercellular communication and the control of tissue growth: lack of communication between cancer cells. Nature, 1966. 209(5029): p. 12489.

4. Zhu, D., et al., Growth retardation in glioma cells cocultured with cells overexpressing a gap junction protein. Proc Natl Acad Sci U S A, 1992. 89(21): p. 10218-21.

5. Goldberg, G.S., K.D. Martyn, and A.F. Lau, A connexin 43 antisense vector reduces the ability of normal cells to inhibit the foci formation of transformed cells. Mol Carcinog, 1994. 11(2): p. 106-14.

6. Lampe, P.D. and A.F. Lau, The effects of connexin phosphorylation on gap junctional communication. Int J Biochem Cell Biol, 2004. 36(7): p. 1171-86. 
7. Tomasetto, C., et al., Specificity of gap junction communication among human mammary cells and connexin transfectants in culture. J Cell Biol, 1993. 122(1): p. 157-67.

8. Gakhar, G., D. Schrempp, and T.A. Nguyen, Regulation of gap junctional intercellular communication by TCDD in HMEC and MCF-7 breast cancer cells. Toxicol Appl Pharmacol, 2008.

9. Kanczuga-Koda, L., et al., Connexins 26 and 43 correlate with Bak, but not with Bcl-2 protein in breast cancer. Oncol Rep, 2005. 14(2): p. 325-9.

10. Laird, D.W., et al., Deficiency of connexin43 gap junctions is an independent marker for breast tumors. Cancer Res, 1999. 59(16): p. 4104-10.

11. Lee, S.W., et al., Transcriptional downregulation of gap-junction proteins blocks junctional communication in human mammary tumor cell lines. J Cell Biol, 1992. 118(5): p. 1213-21.

12. Hirschi, K.K., et al., Gap junction genes $C \times 26$ and $C x 43$ individually suppress the cancer phenotype of human mammary carcinoma cells and restore differentiation potential. Cell Growth Differ, 1996. 7(7): p. 861-70.

13. Huang, R.P., et al., Connexin 43 (cx43) enhances chemotherapy-induced apoptosis in human glioblastoma cells. Int J Cancer, 2001. 92(1): p. 130-8.

14. Gunjan Gakhar, T.O., Aibin Shi, Duy H. Hua, and Thu Annelise Nguyen, Antitumor Effect of Substituted Quinolines in Breast Cancer Cells. Drug Development Research, 2008. 69: p. 526-534.

15. Na, H.K., et al., Restoration of gap junctional intercellular communication by caffeic acid phenethyl ester (CAPE) in a ras-transformed rat liver epithelial cell line. Cancer Lett, 2000. 157(1): p. 31-8.

16. Trosko, J.E. and C.C. Chang, Mechanism of up-regulated gap junctional intercellular communication during chemoprevention and chemotherapy of cancer. Mutat Res, 2001. 480-481: p. 219-29.

17. Shi, A., et al., Synthesis and anti-breast cancer activities of substituted quinolines. Bioorg Med Chem Lett, 2008. 18(11): p. 3364-8.

18. Jemal, A., et al., Cancer statistics, 2009. CA Cancer J Clin, 2009. 59(4): p. 225-49.

19. Lampe, P.D., et al., Phosphorylation of connexin43 on serine368 by protein kinase $C$ regulates gap junctional communication. J Cell Biol, 2000. 149(7): p. 1503-12.

20. Heiniger, B.G., G.; Prasain, K.; Hua, D. H.; Nguyen, T. A., Second Generation of Substituted Quinolines as Anti-Cancer Drugs for Breast Cancer. Anticancer Res, 2010. 30 (10).

21. Plobeck, N.; Delorme, D.; Wi, Z.-Y.; and others. (2000) New diarylmethylpiperazines as potent and selective nonpeptidic $d$ opioid receptor agonists with increased in vitro metabolic stability. J. Med. Chem. 43, 3878-3894. 